\title{
洞穴鱼类眼部退化机制的研究进展
}

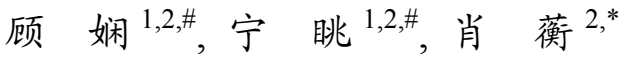

(1. 云南大学 云南省生物资源保护与利用重点实验室, 云南 昆明 650091; 2. 云南大学 生命科学学院, 云南 昆明 650091)

摘要：黑暗环境中的洞穴鱼类眼部结构发生了退化，但不同洞穴鱼物种眼部退化程度存在差异，从眼部结构 的部分缺失到完全消失的情况均存在。目前研究表明, 不论是达尔文的自然选择学说, 还是木村资生的中性进化 理论均不能很好地解释洞穴鱼类眼部退化的产生机制。洞穴鱼类眼部退化是一个复杂的过程, 若要揭示其机制需 汇集多个学科的研究优势。该文介绍了国内外洞穴鱼类眼部退化研究领域的形态解剖学、发育生物学、动物行为 学及分子遗传学研究进展, 并对洞穴鱼眼部退化的研究现状与发展提出了一些思考和建议。

关键词: 洞穴鱼类; 眼部; 退化

中图分类号：Q959.499; Q951;Q958.8 文献标志码：A＼cjkstart文章编号：0254-5853-(2012)04-0409-07

\section{Progress on the degeneration mechanism of cave fishes' eyes}

\author{
GU Xian ${ }^{1,2, \#}$, NING Tiao ${ }^{1,2, \#}$, XIAO Heng ${ }^{2, *}$ \\ (1. Laboratory for Conservation and Utilization of Bio-Resources, Yunnan University, Kunming 650091, China; \\ 2. School of Life Sciences, Yunnan University, Kunming 650091, China)
}

\begin{abstract}
Attempts to understand the degeneration of the eyes in cave fish has largely been explained by either various extents of gradual degeneration, ranging from partial to total loss, observed in various species or by acceleration of loss caused by dark environments. However, neither the theory of biological evolution developed by Charles Darwin nor the neutral theory of molecular evolution formulated by Kimura Motoo adequately explains these phenomena. Recent trends in utilizing multidisciplinary research, however, have yielded better results, helping reveal a more complex picture of the mechanisms of degeneration. Here, we summarize the current progress of the research via morphology and anatomy, development biology, animal behavior science and molecular genetics, and offer some perspectives on the ongoing research into the development and degeneration of eyes in cave fish.
\end{abstract}

Key words: Cave fishes; Eyes; Degeneration

十八世纪中期, Schiodte (1849)首次对洞穴生物 进行了生态学划分, 从而拉开了洞穴生物研究的序 幕。洞穴鱼是一类重要的洞穴生物。“洞穴鱼类”广 义上指生活在洞穴以及类似洞穴环境下的鱼形动 物; 狭义则指陆地生态系统中生活在常年有水的岩 溶洞穴以及地下河、湖等生态环境中的鱼类 (Zhao \& Zhang, 2009)。本文主要探讨生活于洞穴或地下 水环境中, 并表现出适应性特征的狭义洞穴鱼类。 最新统计数据表明, 全世界目前有记述的洞穴鱼类
共 122 种, 分属 10 目(鲤形目、脂鲤目、鲇形目、 电鳗目、鲑鲈目、鼠由鳚目、鳞形目、合鳃鱼目、鲈 形目和鲉形目)19 科 53 属, 其中鲤形目的洞穴鱼种 类最丰富(Zhao \& Zhang, 2009)。鲤形目洞穴鱼主要 分布于中国西南及泰国等地区。鲇形目、脂鲤目等 其他洞穴鱼主要分布在巴西、墨西哥、马达加斯加 等热带国家和地区。

洞穴鱼类通常由于体表色素与鳞片的消失而 呈现半透明状的身体特征, 从而引起人们的关注及

\footnotetext{
收稿日期: 2011-12-26; 接受日期: 2012-06-25

基金项目：云南大学第三届研究生科研课题项目(YNUY201035); 国家 “973” 项目(2007CB411600)

*通信作者(Corresponding author), E-mail: xiaoheng@ynu.edu.cn

\#共同第一作者(Authors contributed equally to the work)

第一作者简介: 顾涃(1986-), 女, 硕士研究生, 主要研究方向: 动物学, E-mail: gx.ff@163.com; 宁眺(1978-), 女, 博士, 讲师, 主要研究方向: 动物分子遗传与进化, E-mail: tiaoning@ynu.edu.cn
} 
研究兴趣 (Vandel, 1965)。随着研究的深入, 人们发 现除身体半透明外, 洞穴鱼类往往缺少眼部结构 (即便有残留的眼部结构, 也丧失或基本丧失了辨 色的视雉细胞), 但它们的触须和侧线系统却高度 发达, 有些种类, 甚至演变出一些特殊的感觉结构, 如: 犀角金线鲃(Sinocyclooheilus rhinocerous)等在 头背交界处形成的特殊角状结构。洞穴鱼类的眼部 退化是自然选择的结果, 然而, 无法用经典的达尔 文自然选择学说加以解释, 因为有眼与无眼的洞穴 鱼并非在黑暗环境中均同时存在, 而无眼的洞穴鱼 更适应黑暗环境, 所以“适者生存”下来。真实的情 况是眼睛器官是否存在并不影响洞穴鱼的生存, 洞 穴鱼眼部退化似乎更像是一种“用进废退”现象。 1968 年, 木村资生提出中性突变理论, 认为在分子 水平上, 大多数的物种进化和种内变异是通过那些 对选择呈中性或近中性的等位基因突变的遗传漂 变而非自然选择所引起 (Kimuram, 1968)。然而, 迄 今洞穴鱼视觉相关基因研究并未发现等位基因的 突变, 中性进化理论也未能解释洞穴鱼类眼部退化 的原因。目前, 洞穴鱼类眼部退化机制仍无定论。 这个领域的研究或许会引导我们对目前已有的某 种进化理论进行补充修订, 甚至发现和建立一种新 的物种进化理论。

\section{1 形态解剖学研究进展}

生物学领域的早期研究大多是以描述、记录、 归类的方法对实验材料进行外部形态观察及内部 构造解剖开始。在最初洞穴鱼类眼部退化的形态解 剖学研究中, 科学家们借助低倍光学显微镜观察认 为长期在暗环境中生活的这些鱼类的眼部结构高 度退化且视觉和感光功能完全丧失。随着形态解剖 学研究技术的不断进步, 早期的低倍光学显微镜粗 略观察渐渐被高倍光学显微镜、苂光显微镜、相差 显微镜、电子显微镜等精确观察所取代(Yang, 1992), 观察的对象也由表及里, 深入到细胞器的层面(Liu \& Zheng, 1997)。借助高倍光学显微镜科学家们揭示 了洞穴鱼眼部构造的真实情况，虽然洞穴鱼类眼部 被表皮覆盖, 被脂肪填充 (Nguyen-Legros et al, 1987), 但视网膜仍有残留(Zilles et al, 1983)。随后 的超薄切片显微观察结果指出, 洞穴鱼的视网膜中 没有可辨色的视雉细胞, 但可感光的视杆细胞有所 残留(Kos et al, 2001), 这与正常鱼类的视网膜同时 含有视杆和视锥细胞的情况相异 ( Li \& Tao,
2002)。除依赖残存的视杆细胞进行感光外, 洞穴鱼 还借助松果体中残留的感光细胞对光做出一定反 应。松果体是哺乳动物脑内的一种重要神经内分泌 转换器(Fan et al, 2001)。硬骨鱼的松果体不但具有 内分泌功能, 还具有感光作用, 因此, 又被称作顶 眼或者退化的第三只眼(Wang et al, 1994)。通过高倍 光学显微镜的精细观察发现洞穴鱼类的松果体也 存在不同程度的退化，但显微镜下仍可以分辨出残 存的感光细胞(Besharse \& Hollyfield, 1976; McNulty, 1978)。进一步利用电子显微镜对洞穴鱼与地表硬骨 鱼松果体感光细胞的超微形态结构进行解剖比较 发现，洞穴鱼类松果体中的感光细胞外节的结构与 地表生活的硬骨鱼存在明显区别(Herwig，1976; Kos \& Bulog, 2000)。除了眼部构造的变化，洞穴鱼 类妙面中与眼部相关联的一些骨骼结构, 如围眀骨 系也发生了改变而异于地表的硬骨鱼类(Jeffery, 2008; Yamamoto et al, 2003)。然而，无论是最初通过 光学显微镜看到洞穴鱼退化的眼部被膜覆盖, 还是 后期通过电子显微镜揭示洞穴鱼退化眼部的细胞 组成异常, 以及与眼部感光有关联的嗅球细胞比地 表普通鱼类增多等现象，都是洞穴鱼类眼部退化的 表象观察结果，尚属于定性的形态解剖研究。近几 年, 形态解剖学发展出一种全新的形态计量术。该 技术运用数学原理, 利用图像分析仪对生物组织和 细胞进行二维或三维的形态学测量, 从而使得形态 解剖学研究由定性阶段发展到了定量阶段(Peng, 2009)。形态计量等定量型的新技术的运用必将推动 洞穴鱼类眼部退化的形态解剖学研究向更科学、更 精准的方向发展。

\section{2 发育生物学研究进展}

对于脊椎动物而言, 眼部发育的一个特征是眼 泡的出现(Jeffery, 2005)。随着眼泡的出现, 外肧层 的细胞开始分裂分化，在向心端的位置伸展形成视 柄，另一末端的细胞形成视杯，同时，位于外胚层 的视泡边缘的细胞开始变厚形成晶状体基板 (Jeffery，2005；Yang，2008)。在稍后的发育时期中， 肧胎的每个组织都会从干细胞得到大量高度分化 的细胞，每个干细胞在不对称的分化成一个体细胞 和一个干细胞后会停止分化, 新生的干细胞则继续 这一过程。然而，硬骨鱼的眼部发育稍有不同，其 眼部细胞的生长贯穿细胞的一生, 因而它们的眼睛 才能与身体同比例增长(Durand，1976; Espinasa \& 
Jeffery, 2006)。

显微注射技术是发育生物学中最经典且应用 最广泛的实验技术，该技术可以对肧胎发育过程进 行定位观察, 并且通过显微注射技术可以将外源物 质引入到发育中的胚胎, 研究目的基因的表达对各 个器官的作用(Chen, 1995)。因此, 显微注射技术很 早就被运用到洞穴鱼眼部退化的发育生物学研究 领域。Cahn (1958)比较研究洞穴鱼与地表鱼(地表 鱼是一个相对概念, 指生活于地表水体且表型不具 有洞穴适应特征的鱼类)的胚胎发育过程后提出洞 穴鱼的眼睛是随着身体发育过程而逐渐消失的。

Durand et al (1993)运用显微注射技术对洞穴鱼类胚 胎发育过程进行定位观察, 进一步证实了洞穴鱼类 的眼部退化伴随着身体发育而进行。洞穴鱼并非像 最初设想的那样从胚胎期就不存在眼部构造。真实 情况是, 在胚胎发育初期, 同物种的地表类型和洞 穴类型都遵循正常的眼部发育的过程。经过一段时 期的发育后, 洞穴类型的晶状体细胞最先开始出现 细胞凋亡, 然后细胞调亡在整个眼部组织蔓延。到 洞穴鱼成体发育成熟时, 其眼部已完全被其他迅速 分化发育的组织填充或者包埋(Strickler et al, 2007; Tian \& Price, 2005)。上述研究中观察到洞穴鱼眼部 最早的调亡细胞出现在晶状体中, 这一现象提示晶 状体可能是控制眼部发育和退化的重要构件。通过 显微注射结合晶状体移植手术, 研究者互换处在相 同发育时期的同物种的地表类型和洞穴类型的晶 状体, 洞穴类型能发育出正常的眼部表型, 而地表 类型眼部细胞开始调亡。若在地表类型的眼部开始 出现细胞调亡时, 再将原来的晶状体换回来, 则地 表类型的眼部发育又恢复。这一结果证实了晶状体 是控制眼部发育和退化的重要构件(Jeffery, 2005, 2009; Strickler et al, 2007)。然而, 晶状体并非是控 制眼部细胞调亡的唯一控件, 研究显示晶状体可能 是通过与视网膜色素上皮的相互作用来调节眼部 调亡的进程(Strickler et al, 2007)。

\section{3 行为学研究进展}

动物行为学研究动物对环境的应激性以及动 物间的沟通、社交、学习、繁殖、情绪表达等(Shang, 1998，2005)。长期生活于暗环境的洞穴鱼类, 其行 为表现明显异于地表鱼类, 已经发展出完全适应黑 暗环境的受食、攻击、繁殖等行为。由于洞穴鱼类 实验材料的特殊性, 如生境不易发现和进入, 以及
活体样本极难在实验条件下成活等因素，使得洞穴 鱼类眼部退化的行为学研究十分困难。目前仅有简 单光线刺激下洞穴鱼行为表现的少量报道。科学家 研究指出, 在同样黑暗模拟环境中, 地表鱼类显得 更加好斗和不安; 而同样光照模拟环境中, 洞穴鱼 类成体却没有强烈的行为改变(Alunni et al, 2007)。 更有意思的是阴影反应实验中, 洞穴鱼幼体的反应 行为模式更接近于两栖动物幼体而异于硬骨鱼类 (Yoshizawa \& Jeffery, 2008), 且洞穴鱼幼体比成体 对光线的敏感度更强, 洞穴鱼对光环境的应激行为 似乎呈现出随着身体成熟度增加而逐渐减弱消失 的趋势(Espinasa et al, 2005; Kos et al, 2001)。行为学 的研究结果从另一方面支持了发育生物学研究得 出的结论一一洞穴鱼类的眼部退化是伴随身体发 育进行的。除了洞穴鱼个体成熟度不同对光环境的 反应呈现差异外, 洞穴鱼对光源类型及光源照射位 置的反应也不同。洞穴鱼类对集中型光源的应激反 应更快(Strickler et al, 2007), 尤其当光线直接照射 在其大脑松果体所在的位置(Yoshizawa \& Jeffery, 2008)。然而，洞穴鱼类对光源刺激的行为模式、产 生机理以及复杂实验环境刺激下的行为表现及神 经传导关联性等较深层次的行为学研究尚未开展。 因此, 洞穴鱼眼部退化与行为改变间到底遵从哪种 发展关系: 是眼部退化导致了洞穴鱼的行为改变, 还是行为改变加速了眼部退化的进程, 抑或是眼部 退化与行为改变一直相互影响相互作用。至今这些 问题仍是未解之谜。

\section{4 分子遗传学研究进展}

分子遗传学是在分子的结构、功能及相互关系 的基础上来研究生物遗传与变异机制的遗传学分 支学科(Luo, 1997)。分子遗传学的发展依赖于分子 生物学实验技术的不断进步。近 30 年, 分子生物学 实验技术发展非常迅速，因而分子遗传学成为了洞 穴鱼类视觉退化研究中起步最晚而发展最快的方 向。根据分子遗传学理论, 洞穴鱼视觉相关基因的 功能改变或表达通路问题很可能是引起洞穴鱼视 觉退化的关键。因此, 最初洞穴鱼视觉退化的分子 遗传学研究重点是比较洞穴鱼与地表鱼在视觉相 关基因上的序列差异, 找到视觉相关基因的蛋白质 编码区或表达调控区的突变位点。通过对比墨西哥 丽脂鲤(Astyanax mexicanus)的地表类群和洞穴类群 的几种视觉相关基因的分子序列, 发现两个群体中 
相同基因的蛋白质编码区并无变异(Yokoyama \& Yokoyama，1990), 而将两个群体的晶状体蛋白、 pax6、 Rx、Chx、Vax 等对眼部有重要作用的基因 的 cDNA序列与斑马鱼(Brachydanio rerio)等其他鱼 类的相同基因的 cDNA 序列进行比较时, 发现这些 基因 cDNA 序列的鱼类特定区域是同源性的。这说 明洞穴鱼眼部退化并不遵循中性进化。因为中性进 化理论认为中性突变对生物的生存和繁殖没有影 响, 自然选择对它们不起作用, 突变在种群中的保 存、扩散、消失完全随机。加之洞穴鱼的群体通常 都很小, 如果其视觉退化符合中性进化理论, 那么 视觉相关基因的漂变速度应该很快, 就会在 1 2 代 后出现某些基因的固定和另一些基因的消失。而实 际研究情况是洞穴鱼与其它鱼类的视觉相关基因 的 cDNA 序列的鱼类特定区域高度同源。于是洞穴 鱼类分子遗传学研究的重点开始转向眼部发育相 关基因的表达模式，包括表达的位点变化、表达的 时间不同以及表达量的多少。在表达研究中这几种 视觉相关基因在墨西哥丽脂鲤地表与洞穴群体中 的表达方式呈现出差异 (Behrens et al, 1998; Strickler et al, 2002; Tian et al, 2005)。除了视觉相关 基因的表达模式的研究之外, 基因链锁图谱成为洞 穴鱼类分子遗传学研究的另一个新方向。国外研究 者通过分析比较墨西哥丽脂鲤的洞穴类型和地表 类型杂交后代的基因链锁图谱，提出洞穴鱼的眼部 退化是多基因作用的过程 (Strickler \& Jeffery, 2009)。

目前洞穴鱼视觉研究主要集中在对眼部发育 有重要作用的以下几个基因或基因家族中。1)最先 引起人们的注意是 Hedgehog ( $h h$ ) 信号基因。 Hedgehog 信号能够调控多种器官的发育。 $h h$ 信号 基因是 Hammerschmidt 等人首先在果蝇肧胎中发现 的控制信号通路的基因(Wang et al, 2011)。该信号基 因在硬骨鱼中有两个同源基因： $s h h$ 和 $t w h h$ (Ekker et al, 1995), 它们有重叠的表达模式。与地表鱼不同 的是，许多洞穴鱼的这两个基因在胚胎中线位置的 表达区域都有一个扩充的现象。如果对地表鱼的肧 胎注射这两个基因的 mRNA, 成体地表鱼的眼部会 消失, 这说明这两个基因的扩充表达对眼部发育有 阻碍的作用。同时发现在洞穴鱼晶状体的凋亡过程 中 $s h h$ 信号基因的作用更为重要(Yamamoto et al, 2004)。2)Pax 基因家族。该基因家族受 $h h$ 信号基 因调控, 编码 128 个氨基酸且在进化上保守(Pan \&
Yuan, 1997), 其表达的蛋白质对动物肧胎时期的器 官发育有重要作用。家族中的 pax6 对眼部发育作 用很大(An et al, 2005; Liu et al, 2004)。通过对比洞 穴鱼的表达模式发现，与 $h h$ 基因不同, pax6 的表达 较为缓和(Behrens et al, 1997; Strickler et al, 2001), 即在表达位置的表达程度有所减弱。3)Prox1 基因。 由于该基因在许多器官都有表达，所以人们推测这 个基因可能也受到 $h h$ 信号基因的调控。在金鱼的 prox1 表达研究中，人们发现不同发育时期金鱼眼 部该基因的表达模式有所不同(Jeffery et al, 2000; Ma et al, 2007)，提示 proxl 基因在不同发育时期的 作用可能不同。Proxl 基因在洞穴鱼的嗅球和神经 丘的表达有所扩充，但在眼部发育中该基因的表达 是正常的, 这说明在嗅球等器官上 prox 1 有重要作 用。4)Hsp 热休克蛋白。该蛋白对于处于不良环境 下的生物体细胞具有保护作用(Sun, 1997), 且按相 对分子质量大小分为 hsp90、hsp70、hsp60 及小 hsp 四个家族(Morimoto et al, 1990)。Hsp70 在同种鱼类 的洞穴类型和地表类型的晶状体中的表达模式相 同，而 hsp90 编码的一种 hsp90 亚型在两者的晶状 体中没有表达，另一种 hsp $90 \alpha$ 亚型只在洞穴群体的 晶状体中表达且在晶状体调亡之前会出现一个表 达高峰，提示该亚型在洞穴鱼的晶状体调亡的过程 中可能具有重要作用(Hooven et al, 2004)。

\section{5 总结与展望}

洞穴鱼的奇异外形引起了人们极大的兴趣，从 中世纪开始就有相关的记录资料。然而，由于洞穴 环境复杂，采样非常困难，使得洞穴鱼的研究发展 缓慢。同时洞穴鱼类很难像其他鱼类一样人工繁殖 和驯养, 这无疑加大了研究的困难性。目前仅有墨 西哥丽脂鲤一种洞穴鱼被成功驯化成观赏鱼类, 其 余洞穴鱼多被列为保护物种。这也是限制洞穴鱼研 究的另一个重要因素。即便在生物技术蓬勃发展的 21 世纪，洞穴鱼的研究多数仍集中在样品易获取的 墨西哥丽脂鲤的发育学、肧胎学、组织解剖学，以 及少数洞穴鱼，如金线鲃等的系统发育学上。洞穴 鱼类的进化是一个复杂的过程, 需要系统性研究。 然而，目前尚无任何一个进化学说能够很好解释洞 穴鱼类的进化及其眼部的退化。开展洞穴鱼类眼部 退化机制的研究，能够让研究者更深刻的理解动物 的表型进化与基因之间的联系，或许能够促进某种 旧进化理论的修订以及一个新进化理论的建立。中 
国拥有全世界最丰富的洞穴鱼类资源，充分利用和 保护好资源优势, 紧跟世界进化研究的步伐, 我国 科研工作者便可在洞穴鱼类眼部退化的研究中有 所成就。针对目前洞穴鱼类研究现状, 笔者提出今 后洞穴鱼类研究中需要注意的以下几方面内容。

\section{1 发展采样手段, 丰富研究材料}

鱼类采集的常规方法很多，如竿钓、撒网、电 击等。通常这些方法只适合于在水域较宽的环境中 使用，而且对样品具有一定损伤。由于洞穴环境复 杂特殊, 且洞穴鱼生命力较脆弱, 上述方法并不适 用于洞穴鱼的采集。为了获得丰富的洞穴鱼研究材 料, 可以根据洞穴鱼的以下特点, 发展切实可行的 采样手段。首先, 雨季时期洞穴鱼类会随地下暗河 水位升高而进入相邻的洞穴水域, 这是采集洞穴鱼 的最佳时期。在这个时期进入洞穴可以增加采样成 功几率。其次, 洞穴地下河中往往食物稀缺, 设置 地笼并辅以食物诱捕有一定效果。再者, 洞穴鱼类 对声波及光线都比较敏感, 通过声波或光线刺激将 鱼类向目标渔网中驱赶或引诱, 也能提高采样效 率。总之, 洞穴鱼的采样方法需结合洞穴鱼的生物 特性而设计, 这样才能无损伤且有效地增加采样量, 丰富研究材料。

\section{2 跨学科研究，多种技术结合}

1904 年，洞穴生物学这个概念首次被提出 (Protas et al, 2008)。从此揭开了洞穴鱼类的研究历 史。悉数以前的研究成果, 从最早的形态学和生态 环境研究到后来的系统分类学研究, 都是单一学科 的研究手段。单一研究往往无法解决洞穴鱼类眼部 退化机制这类复杂的生物学问题。要透彻揭示相关 基因在洞穴鱼类的眼部退化中的作用, 跨学科的研 究及多种技术的运用是必经之路。相对于其他学科 的研究技术而言, 分子遗传学研究具有对材料的需 求量较少且 DNA 水平的分析不受限于活体样本的 优点。加之 DNA 测序技术近几年飞速发展, 尤其是 第二代及第三代高通量测序技术的发明和运用使 得生物体遗传信息的获取速度和准确性大幅度提 升(Sultan et al; Hayden, 2009; Shendure \& Ji, 2008)。 因而运用高通量测序新技术获取洞穴鱼基因组的
分子遗传信息(目前尚无任何一种洞穴鱼基因组数 据公布)并从中篎选出与眼部退化相关的目标基因 进行深入分析是探讨洞穴鱼类眼部退化机制的一 个突破口。同时，建立以形态学为基础，生态学为 导向, 胚胎发育及神经生物学为验证的跨学科多技 术结合策略，必将加速洞穴鱼类眼部退化机制领域 的研究进程。

\section{3 注重环境保护，攻克人工饲养}

洞穴生境能够孕育很多我们未知的物种，但是 由于洞穴环境的特殊性，很多研究者未曾涉足的洞 穴已经开发成旅游和探险项目。随着大量的游客涌 入，全天候的灯光照明，严重的噪声污染，使得洞 穴环境受到严重破坏，而且这种破坏不可恢复，其 中生存的很多物种很可能在人们未了解前就消失。 这也是几乎所有已发现的洞穴鱼类都被列入濒危 物种的原因之一。加强洞穴环境保护无疑是避免洞 穴鱼类灭绝最直接的措施。然而，目前我国洞穴环 境保护的实施推广异常困难, 且能保护的地区非常 有限。因此，攻克洞穴鱼的人工饲养才能长远有效 地保护更多的洞穴鱼资源。人工饲养不但有益于保 护洞穴鱼，解决洞穴鱼科研上的样品问题，而且利 于构建健康生态系统(例如, 现已成功构建的能有 效改善滇池水体环境的“花(海菜花)一一鱼(滇池金 线鲃)一一蚌(无齿蚌)湿地生态系统”, 创造特色水 产养殖效益。我国科研工作者自 2003 年就开始摸 索滇池金线鲃(Sinocyclocheilus grahami)、犀角金线 鲃 (Sinocyclocheilus rhinocerous)、大头金线鲃 (Sinocyclocheilus macrocephalus)、尖头金线鲃 (Sinocyclocheilus oxycephalus)、抚仙金线鲃 (Sinocyclocheilus tingi)等洞穴鱼种的人工繁育技 术。通过模拟洞穴鱼天然生活环境并利用生物学技 术阻止人工饲养下亲鱼性腺退化的措施，上述几种 洞穴鱼的人工饲养现已获得初步成功(Li et al, 2009; Pan et al, 2009; Yan et al, 2010; Yang et al, 2007)。我 们坚信不久的将来洞穴鱼人工饲养能完全被攻克。 一旦洞穴鱼人工饲养获得成功，经济价值得以体现， 就能更好地促进洞穴鱼的保护。

An F, Guan XK, Huang H. 2005. Discussion that PAX6 acts on while the development take place in the eyes [J]. Tibet Sci Tech, 150(10): 44-45. [安芳, 关小康, 黄鹤. 2005. PAX6 在眼发生发育过程中作用的探讨.
Developmental mechanisms for retinal degeneration in the blind cavefish Astyanax mexicanus [J]. J Comp Neurol, 505(2): 221-233. 
西藏科技, 150(10): 44-45.]

Behrens M, Langecker TG, Wilkens H, Schmale H. 1997. Comparative analysis of Pax-6 sequence and expression in the eye development of the blind cave fish Astyanax fasciatus and its epigean conspecific [J]. Mol Biol Evol, 14(3): 299-308.

Behrens M, Wilkens H, Schmale H. 1998. Cloning of the $\alpha$ A-crystallin genes of a blind cave form and the epigean form of Astyanax fasciatus: a comparative analysis of structure, expression and evolutionary conservation [J]. Gene, 216(2): 319-326.

Besharse JC, Hollyfield JG. 1976. Renewal of normal and degenerating photoreceptor outer segments in the Ozark cave salamander [J]. $J$ Exp Zool, 198(3): 287-301.

Cahn PH. 1958. Comparative optic development in Astyanax mexicanus and two of its blind cave derivatives [J]. Bull Am Mus Nat Hist, 115: $75-112$

Chen YZ. 1995. Molecular Neurobiology [M]. Beijing: People's Military Medical Press. [陈宜张. 1995. 分子神经生物学. 北京: 人民军医 出版社.]

Durand JP. 1976. Ocular development and involution in the European cave salamander, Proteus anguinus laurenti [J]. Biol Bull, 151(3): 450-466.

Durand JP, Keller N, Renard G, Thorn R, Pouliquen Y. 1993. Residual cornea and the degenerate eye of the cryptophthalmic Typhlotriton spelaeus [J]. Cornea, 12(5): 437-447.

Ekker SC, Ungar AR, Greenstein P, von Kessler DP, Porter JA, Moon RT, Beachy PA. 1995. Patterning activities of vertebrate hedgehog proteins in the developing eye and brain [J]. Curr Biol, 5(8): 944-955.

Espinasa L, Jeffery WR. 2006. Conservation of retinal circadian rhythms during cavefish eye degeneration [J]. Evol Dev, 8(1): 16-22.

Espinasa L, Yamamoto Y, Jeffery WR. 2005. Non-optical releasers for aggressive behavior in blind and blinded Astyanax (Teleostei, Characidae) [J]. Behav Processes, 70(2): 144-148.

Fan YB, Hou GQ, Wang BZ. 2001. The mammalian pineal gland: Structure and function [J]. Chn J Neuroanat, 17(1): 91-94. [焚宇兵, 侯广棋, 王保芝. 2001. 哺乳动物松果体的构造和功能. 神经解剖学杂志, 17(1): 91-94.]

Hayden EC. 2009. Genome sequencing: the third generation [J]. Nature, 457(12): 768-769.

Herwig HJ. 1976. Comparative ultrastructural investigations of the pineal organ of the blind cave fish, Anoptichthys jordani, and its ancestor, the eyed river fish, Astyanax mexicanus [J]. Cell Tissue Res, 167(3): 297-324.

Hooven TA, Yamamoto Y, Jeffery WR. 2004. Blind cavefish and heat shock protein chaperones: a novel role for hsp90 $\alpha$ in lens apoptosis [J]. Int J Dev Biol, 48(8-9): 731-738.

Jeffery WR. 2005. Adaptive evolution of eye degeneration in the Mexican blind cavefish [J]. J Hered, 96(3): 185-196.

Jeffery WR. 2008. Emerging model systems in evo-devo: cavefish and microevolution of development [J]. Evol Dev, 10(3): 265-272.

Jeffery WR. 2009. Evolution and Development in the Cavefish Astyanax [M]. Burlington: Academic Press.

Jeffery WR, Strickler AG, Guiney S, Heyser DG, Tomarev SI. 2000. Prox 1 in eye degeneration and sensory organ compensation during development and evolution of the cavefish Astyanax [J]. Dev Genes Evol, 210(5): 223-230.

Kimura M. 1968. Evolutionary rate at the molecular level [J]. Nature, 217(5129): 624-626.

Kos M, Bulog B. 2000. The ultrastructure of photoreceptor cells in the pineal organ of the blind cave salamander, Proteus anguinus (Amphibia, Urodela) [J]. Pfliigers Arch: Eur J Physiol, 439(7): 175-177.

Kos M, Bulog B, Szél Á, Röhlich P. 2001. Immunocytochemical demonstration of visual pigments in the degenerate retinal and pineal photoreceptors of the blind cave salamander (Proteus anguinus) [J]. Cell Tissue Res, 303(1): 15-25

Li WX, Gao H, Liu HY, Chen H, Li YH. 2009. Artificial propagation of Sinocyclocheilus [J]. Yunnan Agric Sci Technol, (1): 55-57. [李维贤, 高慧, 刘汉云, 陈洪, 李云洪. 2009. 金线鱼繁殖方法. 云南农业科 技, (1): 55-57.]

Li WX, Tao JN. 2002. Local dissection of body of the fishes Sinocyclocheilus rhinocerous [J]. J Yunnan Agric Univ, 17(3): 207-209. [李维贤，陶进能. 2002. 犀角金线鲃的局部解剖. 云南农业大学学 报, 17(3): 207-209.]

Liu LY, Zheng GM. 1997. General Zoology [M]. 3rd ed. Beijing: Higher Education Press. [刘凌云, 郑光美. 1997. 普通动物学. 3 版. 北京: 高等教育出版社.]

Liu XY, Fu T, Wang NL. 2004. Pax6 genes and developmental problems in the eye [J]. For Med Sci :Ophthalmol, 28(4): 217-220. [刘旭阳, 傅 涛，王宁利. 2004. Pax6 基因与眼发育异常. 国外医学:眼科分册, 28(4): 217-220.]

Luo J. 1997. Current situation and prospects of animal molecular genetics [J]. J Yellow Cattle Sci, 23(1): 8-10. [罗军. 1997. 动物分子遗传学 研究现状与展望. 黄牛杂志, 23(1): 8-10.]

Ma DM, Zhu HP, Gui JF. 2007. Molecular cloning and expression characterization of proxl in goldfish (Carassius auratus) eye development [J]. Zool Res, 28(6): 589-596. [马冬梅, 朱华平, 桂建 芳. 2007. 金鱼 Proxl 基因 cDNA 的克隆及其在眼睛发育中的表达 分析. 动物学研究, 28(6): 589-596.]

McNulty JA. 1978. Fine structure of the pineal organ in the troglobytic fish, Typhlichthyes subterraneous (Pisces: Amblyopsidae) [J]. Cell Tissue Res, 195(3): 535-545.

Morimoto RI, Tissières A, Georgopoulos C. 1990. The stress response, function of the proteins, and perspectives [M]// Stress Proteins in Biology and Medicine. New York: CPHL Press 1990:1670-1678.

Nguyen-Legros J, Durand J, Simon A, Keller N, Vigny A, Dupuy J, Pouliquen Y. 1987. Persistence of retinal dopamine cells in the degenerated eye of the cave salamander, Proteus anguinus L. [J]. Ophthalmic Res, 19(6): 309-317.

Pan MH, Yuan JG. 1997. Pax-A development important genes related to family [J]. J Med Mol Biol, 19(2): 49-53. [潘美辉, 袁建刚. 1997. Pax- 一个发育相关的重要基因家族. 医学分子生物杂志，19(2): 49-53.]

Pan XF, Liu SW, Li ZY, Yang JX. 2009. Artificial propagation and larvae cultivation of Sinocyclocheilus tingi [J]. Zool Res, 30(4): 463-467. [潘晓赋, 刘淑伟, 李再云, 杨君兴. 2009. 抚仙金线鲃人工繁殖与 鱼苗培育技术. 动物学研究, 30(4): 463-467.]

Peng KM. 2009. Animal Histology and Embryology [M]. Beijing: Higher Education Press. [彭克美. 2009. 动物组织学及胚胎学. 北京: 高 等教育出版社.]

Protas M, Tabansky I, Conrad M, Gross JB, Vidal O, Tabin CJ, Borowsky R. 2008. Multi-trait evolution in a cave fish, Astyanax mexicanus [J]. Evol Dev, 10(2): 196-209.

Schiodte JC. 1849. Specimen Faunae Subterraneae. Bidrag Til den Underjordiske Fauna [M]. Copenhagen: Kjobenhavn.

Shang YC. 1998. Behavioral Ecology [M]. Beijing: Peking University Press [尚玉昌. 1998. 行为生态学. 北京: 北京大学出版社.]

Shang YC. 2005. Ethology [M]. Beijing: Peking University Press. [尚玉 昌. 2005. 动物行为学. 北京: 北京大学出版社.]

Shendure J, Ji H. 2008. Next-generation DNA sequencing [J]. Nat Biotechnol, 26(10): 1135-1145.

Strickler AG, Famuditimi K, Jeffery WR. 2002. Retinal homeobox genes and the role of cell proliferation in cavefish eye degeneration [J]. Int $J$ Dev Biol, 46(3): 285-294. 
Strickler AG, Jeffery WR. 2009. Differentially expressed genes identified by cross-species microarray in the blind cavefish Astyanax [J]. Int Zool, 4(1): 99-109.

Strickler AG, Yamamoto Y, Jeffery WR. 2001. Early and late changes in Pax6 expression accompany eye degeneration during cavefish development [J]. Dev Genes Evol, 211(3): 138-144.

Strickler AG, Yamamoto Y, Jeffery WR. 2007. The lens controls cell survival in the retina: evidence from the blind cavefish Astyanax [J]. Dev Biol, 311(2): 512-523.

Sultan M, Schulz MH, Richard H, Magen A, Klingenhoff A, Scherf M, Seifert M, Borodina T, Soldatov A, Parkhomchuk D, Schmidt D, O'Keeffe S, Haas S, Vingron M, Lehrach H, Yaspo ML. 2008. A global view of gene activity and alternative splicing by deep sequencing of the human transcriptome [J]. Science, 321(5891): 956-960.

Sun JJ. 1997. The distribution of hsp70, adjust and function [J]. For Med Sci:Pathophysiol Clin Med, 17(1): 7-10. [孙经建. 1997. 热休克蛋白 70 的分布、调节及功能. 国外医学: 生理、病理科学与临床分册, 17(1): 7-10.]

Tian NMML, Price DJ. 2005. Why cavefish are blind [J]. BioEssays, 27(3): 235-238.

Vandel A. 1965. Biospeleology: the Biology of Cavernicolous Animals [M]. Oxford: Pergamon Press.

Wang DQ. 1994. A study on microscopic structure and ultrastructure of the pineal body in a teleost fish (Carassius Auratus) [J]. Acta Hydrobiol $S c i, 18(1): 22-25$. [王典群. 1994. 鲫鱼松果体的显微和超微结构研 究. 水生生物学报, 18(1): 22-25.]

Wang QL, Su L, Liu XG. 2011. Relationship between Hedgehog signaling pathway and related tumors [J]. J Chem Life, 31(1): 21-26. [王琪琳, 苏玲, 刘相国. 2011. Hedgehog 信号通路与肿瘤. 生命的化学, 31(1): 21-26.]

Yamamoto Y, Espinasa L, Stock DW, Jeffery WR. 2003. Development and evolution of craniofacial patterning is mediated by eye-dependent and -independent processes in the cavefish Astyanax [J]. Evol Dev, 5(5): 435-446.

Yamamoto Y, Stock DW, Jeffery WR. 2004. Hedgehog signalling controls eye degeneration in blind cavefish [J]. Nature, 431(7010): 844-847.

Yan H, Tu SH, Dong WH, Yang RL. 2010. Study on the artificial propagation of Sinocyclocheilus graham in Dianchi lake [J]. $J$ Hydrocolloid, 3(1): 138-141. [严晖，涂书浩，董文红，杨瑞林. 2010. 滇池金线鲃全人工繁殖研究. 水生态学杂志, 3(1): 138-141.]

Yang AF. 1992. Vertebrate Zoology $[M] .2^{\text {nd }}$ ed. Beijing: Peking University Press. [杨安峰. 1992. 脊椎动物学. 2 版. 北京: 北京大学出版社.]

Yang JX, Pan XF, Li ZY. 2007. Preliminary report on the successful breeding of the endangered fish Sinocyclocheilus grahami endemic to Dianchi lake [J]. Zool Res, 28(3): 329-331. [杨君兴, 潘晓赋, 李再 云. 2007. 云南滇池濒危特有种滇池金线鲃人工繁殖初报. 动物学 研究, 28(3): 329-331.]

Yang TJ. 2008. Pax6 Expression in the Developmental Eyes of Bufo raddei Strauch and the Histology Features and Functions of Chief Parts of Eyeball in the Bactrian Camels [D]. Master thesis, Lanzhou University, Lanzhou. [杨唐军. 2008. 花背蛤蜍 (Bufo raddei Strauch) 眼发育 过程中 Pax6 的表达及双峰驼 (Camelus bactrianus) 眼的组织学研 究. 兰州大学, 兰州.]

Yokoyama R, Yokoyama S. 1990. Solation, DNA sequence and evolution of a color visual pigment gene of the blind cave fish Astyanax fasciatus [J]. Vision Res, 30(6): 807-816.

Yoshizawa M, Jeffery WR. 2008. Shadow response in the blind cavefish Astyanax reveals conservation of a functional pineal eye [J]. J Exp Biol, 211(3): 292-299.

Zhao YH, Zhang CG. 2009. Endemic Fishes of Sinocyclocheilus (Cypriniformes: Cyprinidae) in China - Species diversity, Cave adaptation, Systematics and Zoogeography [M]. Beijing: China Science Press. [赵亚辉, 张春光. 2009. 中国特有金线鲃属鱼类一一物种多 样性、洞穴适应、系统演化和动物地理. 北京: 科学出版社.]

Zilles K, Tillmann B, Bennemann R. 1983. The development of the eye in Astyanax mexicanus (Characidae, Pisces), its blind cave derivative, Anoptichthys jordani (Characidae, Pisces), and their crossbreds. A scanning- and transmission-electron microscopic study [J]. Cell Tissue Res, 229(2): 423-432. 